\title{
Contraction behaviors of Vorticella sp. stalk investigated using high-speed video camera. I: Nucleation and growth model
}

\author{
Junko Kamiguri ${ }^{1}$, Noriko Tsuchiya ${ }^{1}$, Ruri Hidema ${ }^{1}$, Masatoshi Tachibana ${ }^{1}$, Zenji Yatabe ${ }^{1}$, \\ Masahiko Shoji ${ }^{2}$, Chihiro Hashimoto ${ }^{3}$, Robert Bernard Pansu ${ }^{4}$ and Hideharu Ushiki ${ }^{5}$ \\ ${ }^{1}$ United Graduate School of Agricultural Science, Tokyo University of Agriculture and Technology, 3-5-8 Saiwai-cho, Fuchu-shi, Tokyo \\ 183-8509, Japan \\ ${ }^{2}$ Department of Applied Physics, Tokyo University of Agriculture and Technology, 2-24-16 Nakamachi Koganei-shi, Tokyo 184-8588, \\ Japan \\ ${ }^{3}$ Department of Applied Chemistry and Biotechnology, Niihama National College of Technology, 7-1 Yakumo-Cho, Niihama, Ehime 792- \\ 8580, Japan \\ ${ }^{4}$ Laboratoire de Photophysique et Photochimie Supramoléculaires et Macromoléculaires, UMR 8531 CNRS, D'Alembert Institute, ENS \\ Cachan, 61 av. President Wilson, F-94230 Cachan, France \\ ${ }^{5}$ Institute of Agriculture, Tokyo University of Agriculture and Technology, 3-5-8 Saiwai-cho, Fuchu-shi, Tokyo 183-8509, Japan
}

Received May 17, 2011; accepted November 15, 2011

The contraction process of living Vorticella sp. has been investigated by image processing using a high-speed video camera. In order to express the temporal change in the stalk length resulting from the contraction, a damped spring model and a nucleation and growth model are applied. A double exponential is deduced from a conventional damped spring model, while a stretched exponential is newly proposed from a nucleation and growth model. The stretched exponential function is more suitable for the curve fitting and suggests a more particular contraction mechanism in which the contraction of the stalk begins near the cell body and spreads downwards along the stalk. The index value of the stretched exponential is evaluated in the range from 1 to $\mathbf{2}$ in accordance with the model in which the contraction undergoes through nucleation and growth in a onedimensional space.

Corresponding author: Chihiro Hashimoto, Department of Applied Chemistry and Biotechnology, Niihama National College of Technology, 7-1 Yakumo-Cho, Niihama, Ehime 792-8580, Japan.

e-mail: hashimoto@chem.niihama-nct.ac.jp
Key words: Vorticella sp., image processing, high-speed video camera, damped mass spring system, stretched exponential, nucleation and growth

In a variety of designs to generate movement at cellular and molecular levels, one of the fastest cellular mechanics is found in stalked protozoans such as single-celled Vorticella $\mathrm{sp}^{1-10}$. It has been pointed out that the cell motility of Vorticella $\mathrm{sp}$. is independent of the hydrolysis of adenosine triphosphate (ATP) and could be a convincing natural example of biological actuator. Vorticella sp. consists of a slender stalk (100-500 $\mu \mathrm{m}$ in length and $2-3 \mu \mathrm{m}$ in diameter) and a bell-shaped zooid $(30-60 \mu \mathrm{m}$ in diameter). The stalk is attached to a substrate, and it contracts spontaneously within milliseconds and re-extends in a few seconds. Figure 1 shows a Vorticella sp. used in this report.

The spasmoneme, the organelle placed helically in the stalk, is responsible for the contraction of the stalk, as shown in Figure 2. The spasmoneme (1-2 $\mu \mathrm{m}$ in diameter) consists of bundles of filaments $(2-3 \mathrm{~nm}$ in diameter) and shrinks along the longitudinal direction. The spasmoneme of glycerinated Vorticella sp. contracts when $\mathrm{Ca}^{2+}$ is added 


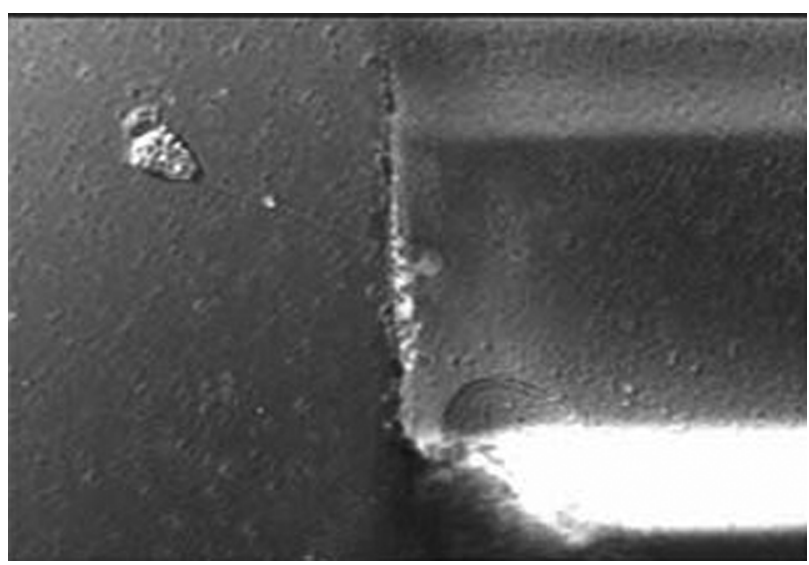

Figure 1 Living Vorticella sp. attached to a glass tube.

and re-extends when $\mathrm{Ca}^{2+}$ is removed using a calcium agent such as EDTA and EGTA ${ }^{1-6}$. It is noted that $\mathrm{Ca}^{2+}$ induces the contraction of spasmoneme and that the hydrolysis of ATP is not necessary for the contraction directly ${ }^{6}$. The contractility of spasmoneme without ATP is fundamentally different from many other types of biological movements such as the contraction of muscles and ciliary motility. Major component of spasmoneme is spasmin, a calcium-binding protein that resemble calmodulin. The contraction could be repeated many times and the presence of membrane tubules inside the spasmoneme is proposed as calcium stores. A tensionextension curve of spasmoneme is described well by the generalized theory of rubber-like elasticity ${ }^{6}$. The observed tension dependence on the concentration of $\mathrm{Ca}^{2+}$ proposed that a smallest unit of spasmoneme generate a tension when being combined cooperatively with about three $\mathrm{Ca}^{2+}$ ions. However, the mechanism of contraction is still not clear at the molecular level.

A few researchers have studied the contraction process of Vorticella sp. in vivo by image processing using a highspeed video camera ${ }^{7-9}$. The motion of a zooid has been considered to be similar to the behavior of a damped harmonic oscillator; in this case, the stalk of the zooid is sub- jected to a Hookean spring forceand the zooid is subjected to a frictional force. The solution of the equation is a double exponential function and very useful to analyze the various experimental data, although a more precise model including the shape of the coiling stalk has been simulated ${ }^{10}$. However, a single exponential instead of a double exponential function was practically applied to the experimental data for the latter stage of the contraction process of Vorticella sp. ${ }^{8,9}$. Here we have attempted to fit the all experimental data in the full range of the contraction process of Vorticella sp. using a double exponential function. It is successful for the contraction process of Vorticella sp. in the aqueous culture media as shown in this report, but it is unsuccessful for the contraction process of Vorticella sp. in the aqueous polymer solution $^{11}$. In addition, a double exponential function is a little bit troublesome in the fitting operation to the data as compared with the following stretched exponential function. Therefore, in this report, a stretched exponential is newly proposed as a useful and simple fitting function to express the entire contraction process of Vorticella sp. and is applied to the data together with a double exponential function.

\section{Experimental}

Vorticella $\mathrm{sp}$. in vivo was obtained from the activated sludge donated by the wastewater treatment plant 'Kitatama Ichigou Water Reclamation Center' (Fuchu-shi, Tokyo, Japan). A small piece of activated sludge was dispersed into the culture solution, that is, an aqueous solution of $\mathrm{KCl}(0.10 \mathrm{M})$, $\mathrm{CaCl}_{2} \cdot 2 \mathrm{H}_{2} \mathrm{O}(0.09 \mathrm{M})$ and $\mathrm{MgSO}_{4} \cdot 7 \mathrm{H}_{2} \mathrm{O}(0.01 \mathrm{M})$. Living cells were taken out by repeating the dispersion procedure and cultured at $21^{\circ} \mathrm{C}$ by adding a few drops of straw juice once a few days. The straw juice was obtained as the filtrate of the mixture of $10-15 \mathrm{~g}$ straws and $1,000 \mathrm{ml}$ water which was boiled for about $20 \mathrm{~min}$ and placed for $1 \mathrm{~d}$, then became opaque due to the Bacillus subtilis. When several glass tubes were set in the culture solution together with living cells in a dish, the cells became attached to the glass tubes within about one week.

The contraction processes of eight Vorticella sp. single

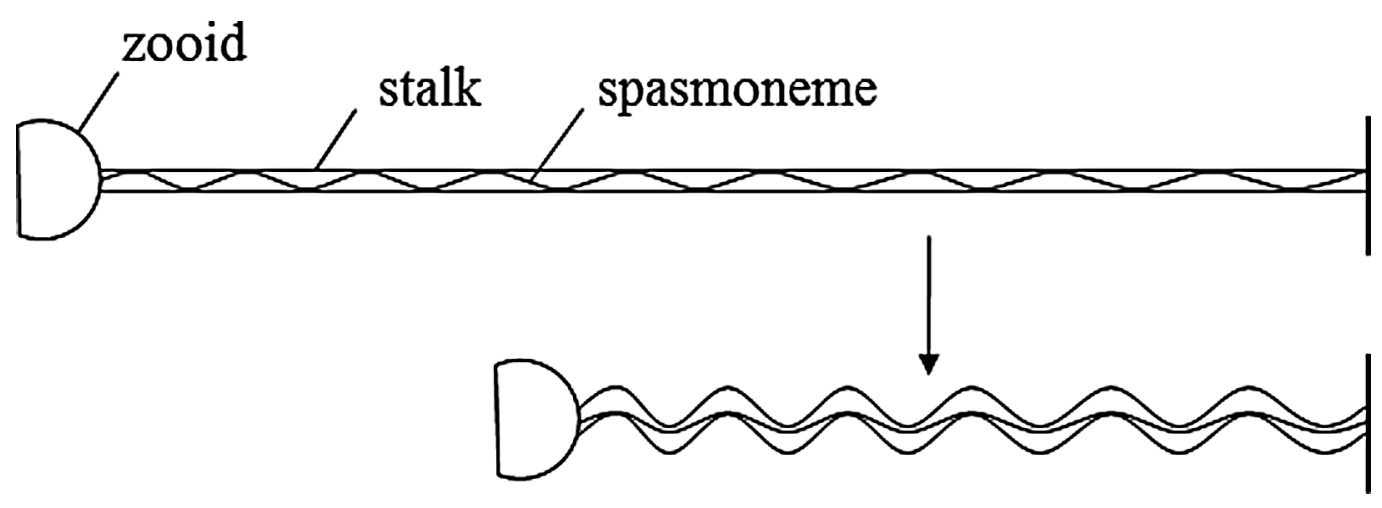

Figure 2 Schematic image of the contraction of Vorticella sp. 
cells attached to glass rods were observed. In order to initiate contraction, the cell was stimulated by hitting the dish. The contraction process was observed using an inverted microscope (IX70; Olympus Co.) and the bright field microscope images were recorded using a high-speed camera system (Ektapro High Gain Imager and Hi-spec Motion Analyzer; Eastman Kodak Co.) at a speed of 6000 frames $/ \mathrm{sec}$. The microscope images were transferred to a personal computer through the video board (Power Capture Pro; Canopus) and processed with Adobe Premiere 6.0 (Adobe Systems Inc.) at a spatial resolution of $640 \times 480$ pixels.

The programs for graphical analysis and curve fitting were developed using Delphi 3.1 (Borland Co., Ltd.). Various fitting functions can be estimated for all data curves by the nonlinear-least-squares method based on the quasiMarquardt algorithm using PLASMA software ${ }^{12,13}$. Calculations were carried out on a personal computer (SF SV2408/B4; Microsoft Windows 2000).

\section{Results and Discussion}

A typical contraction process of Vorticella sp. is shown in Figure 3(a). In Vorticella sp., a bell-shaped zooid is attached to a glass rod through a slender stalk. The stalk is straight at the initial stage. Then, it bends and coils into a helix near the zooid. Subsequently, the contraction spreads to the base of the stalk. The stalk re-extends and returns back to its original length in a few seconds. The coiling of the stalk is observed like a zigzag structure. The turning points of the zigzag structure are named as Top 1, Bottom 1, Top 2, Bottom 2, Top 3, Bottom 3, Top 4, Bottom 4, Top 5 and Bottom 5 from the zooid toward the base of the stalk as shown in Figure 3(b). The sum of the lengths between Top I and Bottom I and the lengths between Bottom I and Top I+1 $(I=1-4)$, that is, the length of the stalk itself does not change on the contraction process, so the turning points can be considered as the markers. The coordinates of these turning points are tracked along $\mathrm{X}$ axis at the origin of the point of attachment of the stalk to the glass. The $\mathrm{X}$ positions of the turning points are plotted as a function of time in Figure 4. The position of Top 1 decreases monotonically, while the position of Top 4 starts to decreases after several milliseconds. It is clear that there is a finite time delay between the motions of the turning points near the zooid and near the base of the stalk. This fact is supported by the experiments about the motion of beads stuck onto the stalk ${ }^{9}$. The fact that the coiling of the stalk starts near the zooid and spreads downwards along the stalk suggests that some stimulus runs down the stalk.

The coordinates of the junction between the zooid and the base of the stalk were tracked to determine the reduced stalk length resulting from the contraction as shown in Figure 5. It takes approximately 15 milliseconds for the stalk of length $460 \mu \mathrm{m}$ to contract. The reduced stalk length, $L_{\text {obs }}(t)$, is plotted as a function of time in Figure 6(a); the length of the fully contracted stalk is assumed to be zero. The instantaneous speed was obtained from the distance travelled during the time interval between frames and is plotted after the Savitzky-Golay seven-point smooth filtering in Figure $6(b)^{14}$. A rapid increase in the speed is observed until it attains a maximum, after which it decays to zero. The following two fitting functions were applied to express the temporal change in the reduced length, $L_{o b s}(t)$, owing to the contraction of Vorticella sp. The goodness of the fitting calculation was evaluated by a value of the residual sum of squares, $\chi^{2}$, as

$$
\chi^{2}=\frac{1}{t_{2}-t_{1}-n} \int_{t_{1}}^{t_{2}}\left(L_{o b s}(t)-L_{c a l}(t)\right)^{2} d t,
$$

where $n, t_{1}$, and $t_{2}$ indicate the number of variable parameters of the fitting function $L_{c a l}(t)$, the initial and the final time of the fitting range, respectively.

\section{A Double Exponential Function -A Damped Spring Model-}

In order to describe the contraction process of Vorticella sp. a damped spring model is applied as follows. The equation of the motion that describes the position of the zooid $L_{c a l}(t)$, as with a damped harmonic oscillator, is

$$
m \frac{d^{2} L_{c a l}(t)}{d t^{2}}=-k L_{c a l}(t)-6 \pi \eta r \frac{d L_{c a l}(t)}{d t},
$$

where $m$ is the mass of the zooid, $r$ is the radius of the zooid, $\eta$ is the viscosity of water, and $k$ is the Hookean force constant of the spring. The contractile force exerted by the Vorticella sp. balances the viscous force on the zooid in Stokes' formula. The solution of Eq. (2) for the over-damped system is a double exponential function as

$$
\begin{aligned}
L_{c a l}(t)= & a \exp \left(-\left(p / 2+\sqrt{p^{2} / 4-q}\right) t\right) \\
& +b \exp \left(-\left(p / 2-\sqrt{p^{2} / 4-q}\right) t\right), \\
& p=\frac{6 \pi \eta r}{m}, q=\frac{k}{m}, \frac{p^{2}}{4}-q>0 .
\end{aligned}
$$

Introducing an initial time delay $t_{0}$, the actual fitting function was used as

$$
\begin{aligned}
L_{c a l}(t)= & a \exp \left(-\left(p / 2+\sqrt{p^{2} / 4-q}\right)\left(t-t_{0}\right)\right) \\
& +b \exp \left(-\left(p / 2-\sqrt{p^{2} / 4-q}\right)\left(t-t_{0}\right)\right), \\
& p=\frac{6 \pi \eta r}{m}, q=\frac{k}{m}, \frac{p^{2}}{4}-q>0 .
\end{aligned}
$$

The $L_{\text {obs }}(t)$ is fitted well by Eq. (4) as shown in Figure 7, although it is a little troublesome to find the initial values of fitting parameters of Eq. (4) as compared with the following stretched exponential function. Figure 7 also shows that only second term of Eq. (4) explains the data in the latter stage of 
(a)

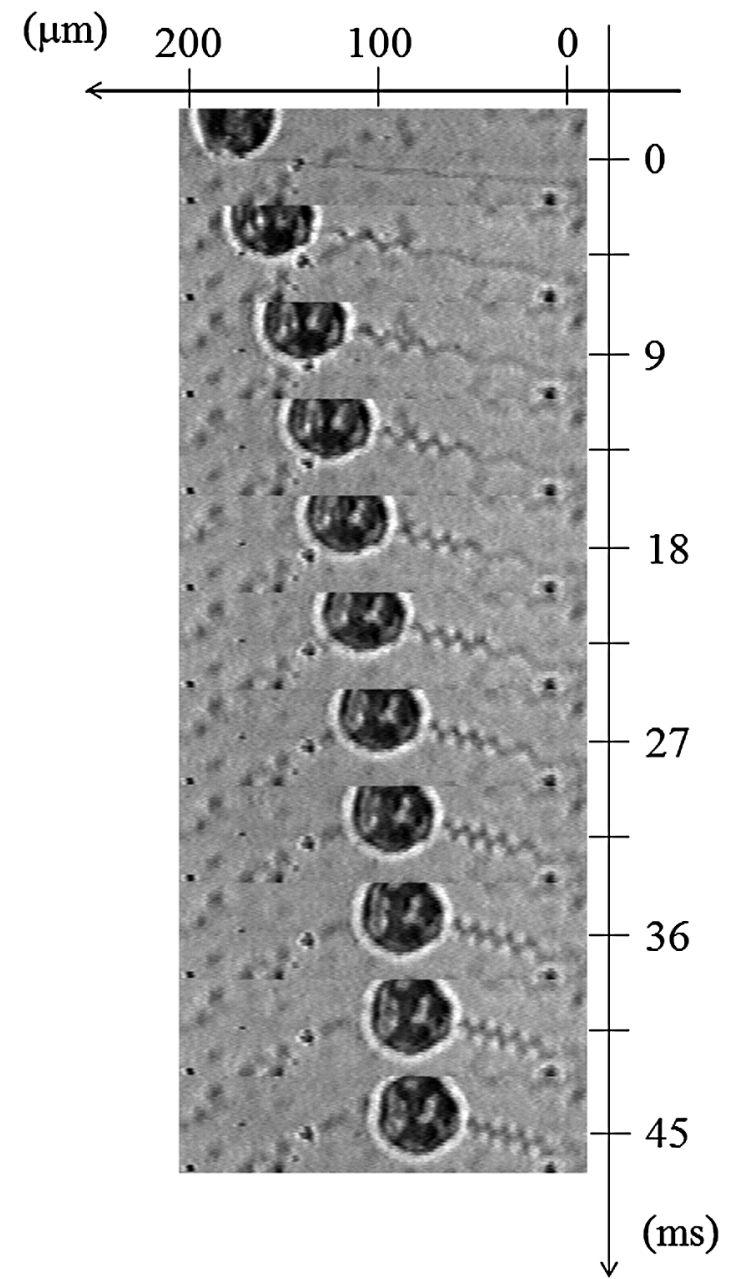

(b)

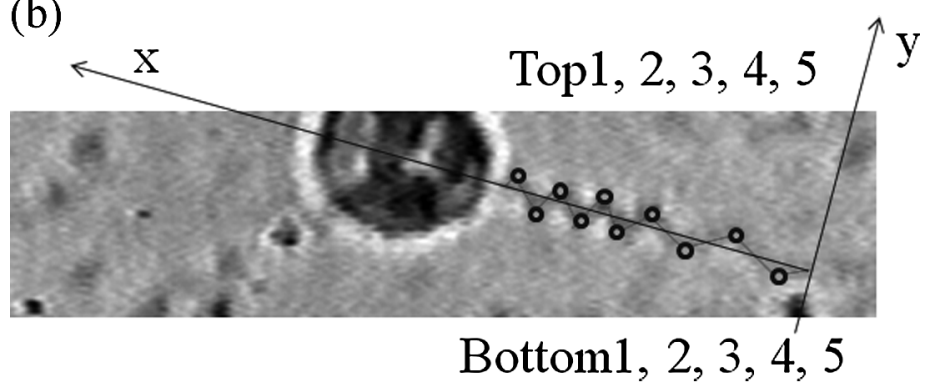

Figure 3 Time series of the contraction of Vorticella sp. (a) and the space coordinates of the turning points of the stalk (b).

the contraction process well. The fitting parameters, $a, b, p$, $q, t_{0}$ and $\chi^{2}$ are listed in Table $1 . \eta$ is $0.890 \times 10^{-3} \mathrm{Ns} / \mathrm{m}^{2}$ and $r$ is $30 \mu \mathrm{m} . \chi^{2}$ is calculated by Eq. (1) with $t_{1}$ as $t_{0}$. Consequently, the mass of the zooid, $m$, and the Hookean force constant, $k$, are evaluated from the value of fitting parameters, $p$ and $q$, respectively. The Hookean force constant, $k$, ranges from $1.1 \times 10^{-4}$ to $1.9 \times 10^{-4} \mathrm{~N} / \mathrm{m}$ (the average over 8 cells: $1.48 \times 10^{-4} \mathrm{~N} / \mathrm{m}$ ) and these values are similar to the results of Y. Moriyama et al. $\left(2.23 \times 10^{-4} \mathrm{~N} / \mathrm{m}\right)^{8}$ and A. Upadhyara et al. $\left(0.6-3.3 \times 10^{-4} \mathrm{~N} / \mathrm{m}\right)^{9}$ for Vorticella con- vallaria. The generated force in the initial stage of the contraction is estimated to be $4.58 \times 10^{-8} \mathrm{~N}$ in average by $F=k x$. This value is consistent with the isometric tension generated by glycerol-treated Vorticella convallaria as pointed by Y. Moriyama ${ }^{8}$. The instantaneous speed of the contraction process is analytically deduced by $u(t)=-d L_{c a l}(t) / d t$ using Eq. (4) as 


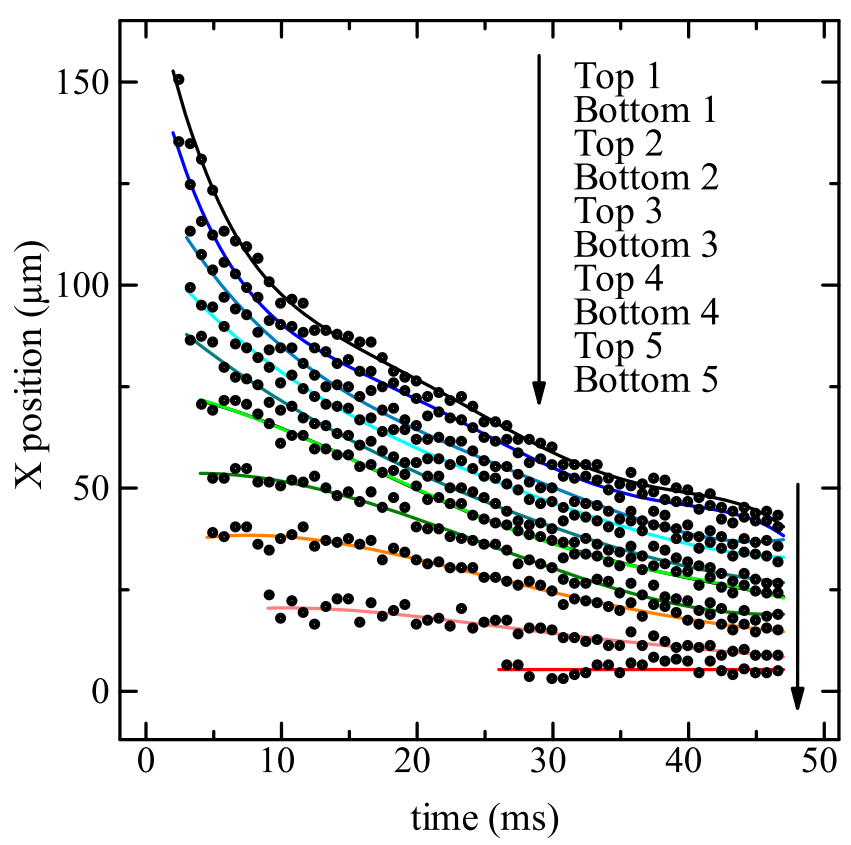

Figure 4 Plots of $X$ position of the turning points along the stalk as a function of time. The values of the $\mathrm{X}$ position are evaluated in a coordinate system of Figure 3(b).

$$
\begin{aligned}
& u(t)= \\
& a\left(p / 2+\sqrt{p^{2} / 4-q}\right) \exp \left(-\left(p / 2+\sqrt{p^{2} / 4-q}\right)\left(t-t_{0}\right)\right) \\
& +b\left(p / 2-\sqrt{p^{2} / 4-q}\right) \exp \left(-\left(p / 2-\sqrt{p^{2} / 4-q}\right)\left(t-t_{0}\right)\right) .
\end{aligned}
$$

The maximum speed of the contraction process, $u_{\max }$, is calculated by

$$
u_{\max }=2 \sqrt{-a b} \sqrt{p^{2} / 4-q}\left\{\sqrt{-a / b}\left|\frac{\sqrt{p^{2} / 4-q}+p / 2}{\sqrt{p^{2} / 4-q}-p / 2}\right|\right\}^{-\frac{p}{2 \sqrt{p^{2} / 4-q}}} .
$$

The evaluated value of $u_{\text {max }}$ ranges from 4.3 to $7.5 \mathrm{~cm} / \mathrm{s}$ for eight cells as listed in Table 1 and the average of $u_{\max }$ is $5.82 \mathrm{~cm} / \mathrm{s}$. This is similar to the results of A. R. Jones for branched Vorticellidae $\left(1.4-5.6 \times 10^{-2} \mathrm{~m} / \mathrm{s}\right)^{6}$, Y. Moriyama et al. $\left(8.8 \times 10^{-2} \mathrm{~m} / \mathrm{s}\right)^{8}$ and A. Upadhyara et al. $\left(5-10 \times 10^{-2} \mathrm{~m} / \mathrm{s}\right)^{9}$ for Vorticella convallaria.

\section{A Stretched Exponential Function}

In order to describe the temporal change of the reduced length resulting from the contraction of the stalk, a stretched exponential function is newly proposed as folloes.

$$
L_{c a l}(t)=c \exp \left(-\left(\frac{t}{\tau_{s}}\right)^{\beta}\right)
$$

where $c$ is the entire reduced length resulting from the con-

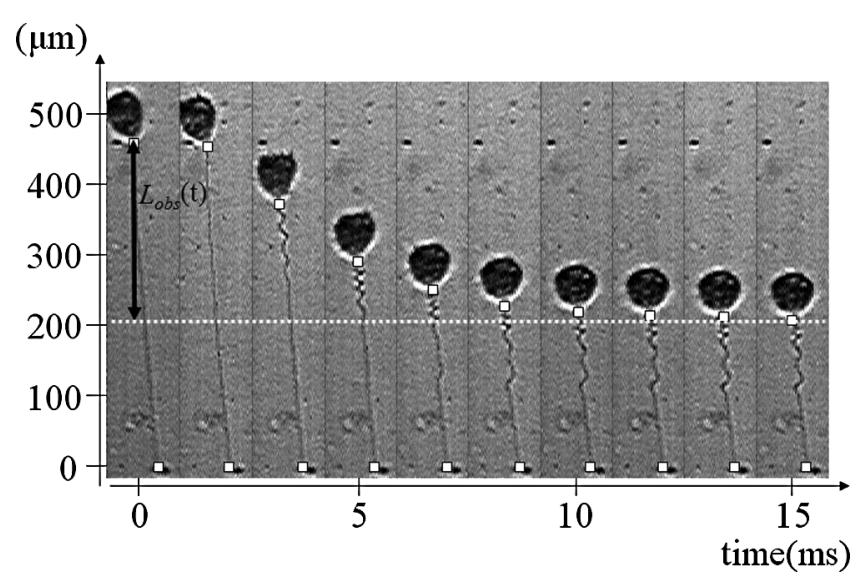

Figure 5 Time series of $L_{o b s}(t)$ on the contraction process of Vorticella sp.

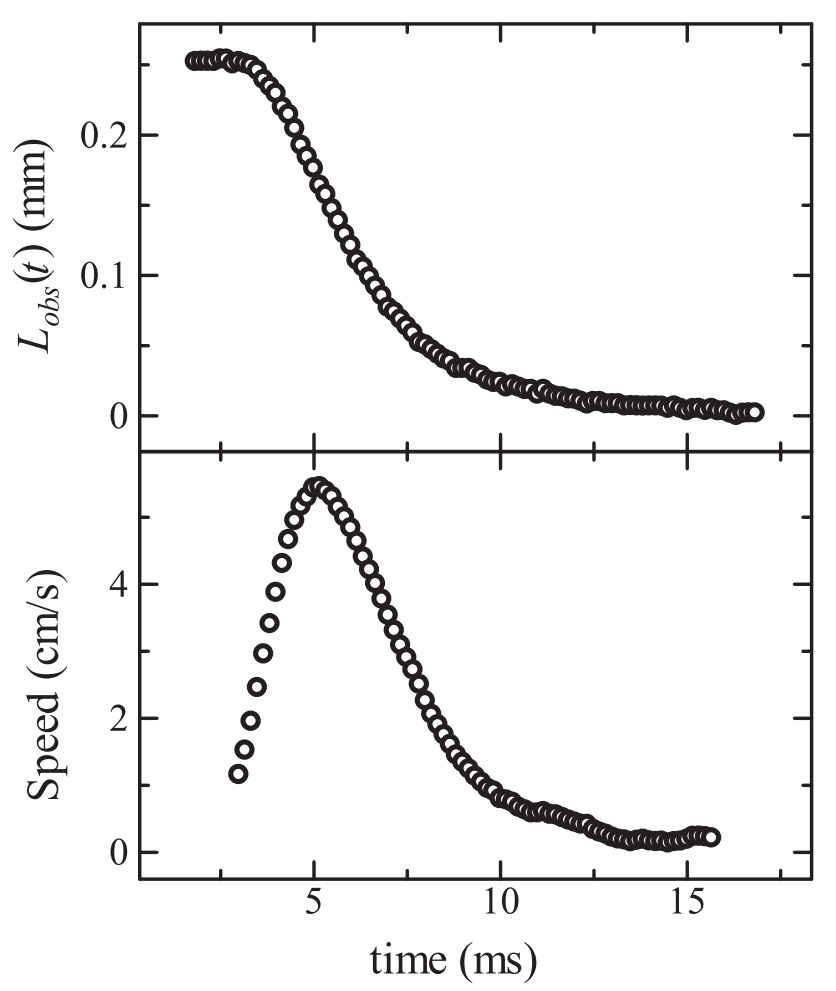

Figure 6 Time courses of $L_{o b s}(t)$ (a) and thecontraction speed (b). The speed data is smoothed by the Savitzky-Golay seven-point filtering.

traction of the stalk, and $\tau_{S}$ is the characteristic time of the contraction. A stretched exponential function (Eq. (7)) with $0 \leq \beta \leq 1$ has been widely used to express the experimental relaxation process in various phenomena and known as Kohlrausch-Williams-Watts (KWW) function ${ }^{15}$. On the other hand, a stretched exponential function (Eq. (7)) with $1 \leq \beta$ was deduced by Avrami to explain the crystallization kinetics ${ }^{16}$ and recently has been discussed from another viewpoint of convolution as well as the case of $0 \leq \beta \leq 1^{16}$. As an actual fitting function, we used 


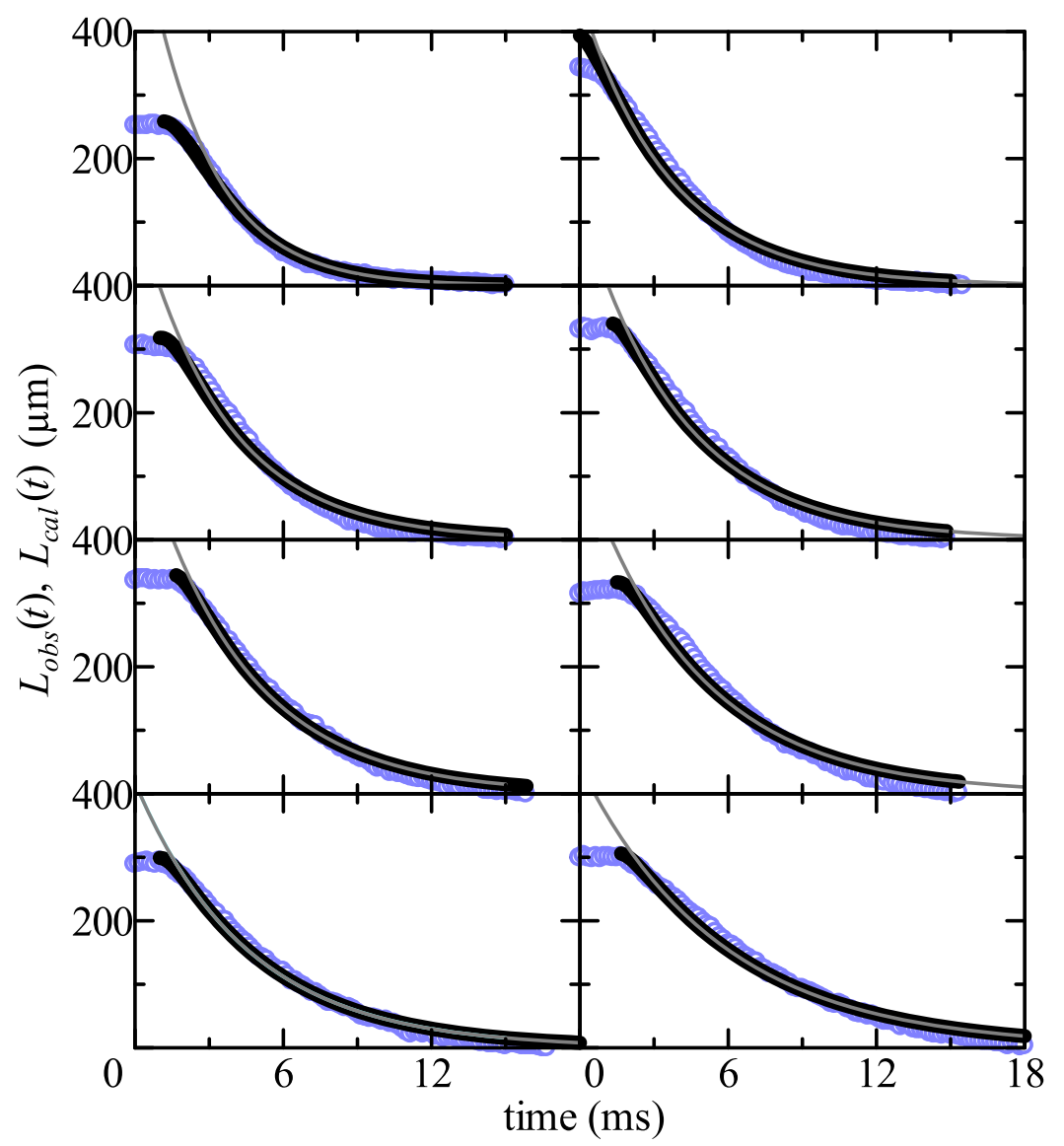

Figure 7 Plots of $L_{o b s}(t)$ as a function of time for several Vorticella sp. The solid black line is a fit to a double exponential function (Eq. (4)) and the gray line means the second term of Eq. (4).

Table 1 Fitting parameters of a double exponential function (Eq. (4))

\begin{tabular}{cccccccccc}
\hline Data & $a(\mu \mathrm{m})$ & $p / 2\left(\mathrm{~ms}^{-1}\right)$ & $q\left(\mathrm{~ms}^{-2}\right)$ & $t_{0}(\mathrm{~ms})$ & $b(\mu \mathrm{m})$ & $\chi^{2}$ & $m(\mu \mathrm{g})$ & $k(\mathrm{~N} / \mathrm{m})$ & $u_{\max }(\mathrm{cm} / \mathrm{s})$ \\
\hline 1 & $-1.39 \times 10^{2}$ & $7.41 \times 10^{-1}$ & $4.24 \times 10^{-1}$ & 1.17 & $3.98 \times 10^{2}$ & $1.7 \times 10^{-5}$ & $4.37 \times 10^{-1}$ & $1.85 \times 10^{-4}$ & 5.68 \\
2 & $-6.27 \times 10$ & $9.34 \times 10^{-1}$ & $4.36 \times 10^{-1}$ & 0.00 & $4.57 \times 10^{2}$ & $2.1 \times 10^{-4}$ & $3.59 \times 10^{-1}$ & $1.57 \times 10^{-4}$ & 7.53 \\
3 & $-7.98 \times 10$ & $8.97 \times 10^{-1}$ & $4.26 \times 10^{-1}$ & 1.00 & $3.98 \times 10^{2}$ & $1.4 \times 10^{-4}$ & $3.61 \times 10^{-1}$ & $1.54 \times 10^{-4}$ & 6.11 \\
4 & $-4.08 \times 10$ & 1.17 & $5.18 \times 10^{-1}$ & 1.33 & $3.81 \times 10^{2}$ & $1.7 \times 10^{-4}$ & $2.84 \times 10^{-1}$ & $1.47 \times 10^{-4}$ & 6.33 \\
5 & $-4.34 \times 10$ & 1.01 & $4.35 \times 10^{-1}$ & 1.67 & $3.88 \times 10^{2}$ & $9.3 \times 10^{-5}$ & $3.45 \times 10^{-1}$ & $1.50 \times 10^{-4}$ & 6.17 \\
6 & $-4.88 \times 10$ & 1.01 & $3.91 \times 10^{-1}$ & 1.50 & $3.82 \times 10^{2}$ & $2.2 \times 10^{-4}$ & $3.50 \times 10^{-1}$ & $1.37 \times 10^{-4}$ & 5.41 \\
7 & $-3.61 \times 10$ & 1.18 & $4.71 \times 10^{-1}$ & 1.00 & $3.35 \times 10^{2}$ & $6.3 \times 10^{-5}$ & $2.96 \times 10^{-1}$ & $1.39 \times 10^{-4}$ & 5.07 \\
8 & $-2.52 \times 10$ & 1.08 & $3.50 \times 10^{-1}$ & 1.67 & $3.31 \times 10^{2}$ & $1.0 \times 10^{-4}$ & $3.17 \times 10^{-1}$ & $1.11 \times 10^{-4}$ & 4.27 \\
\hline
\end{tabular}

$$
L_{c a l}(t)=c \exp \left(-\left(\frac{t-t_{0}{ }^{\prime}}{\tau_{s}}\right)^{\beta}\right)
$$

where $t_{0}{ }^{\prime}$ is an initial time delay. The instantaneous speed of the contraction is analytically deduced by $u(t)=-d L_{c a l}(t) / d t$ using Eq. (8) as

$$
u(t)=c \frac{\beta}{\tau_{s}}\left(\frac{t-t_{0}{ }^{\prime}}{\tau_{s}}\right)^{\beta-1} \exp \left(-\left(\frac{t-t_{0}{ }^{\prime}}{\tau_{s}}\right)^{\beta}\right) .
$$

The maximum speed of the contraction, $u_{\max }$, is also analytically calculated as

$$
u_{\max }=c \frac{\beta}{\tau_{s}}\left(1-\frac{1}{\beta}\right)^{1-\frac{1}{\beta}} \exp \left(\frac{1}{\beta}-1\right) .
$$

$L_{\text {obs }}(t)$ is fitted well by Eq. (8) as shown in Figure 8. Fitting parameters of Eq. (8), $c, \beta, \tau_{S}$ and $t_{0}{ }^{\prime}$ are listed in Table 2 together with the maximum speed, $u_{\max }$, calculated by Eq. (10) and the sum of residue squared value, $\chi^{2}$ calculated by Eq. (1) with $t_{1}$ as $t_{0}{ }^{\prime}$. The value of $\chi^{2}$ of Eq. (8) (a stretched exponential) is $5-22 \times 10^{-6}$ and smaller than that of Eq. (4) (a double exponential), $2-22 \times 10^{-5}$, in spite of that the number of fitting parameters of Eq. (8), 4, is smaller than that of Eq. (4), 5. It indicates that a stretched exponential is more suitable for the fit than a double exponential. In order to dis- 


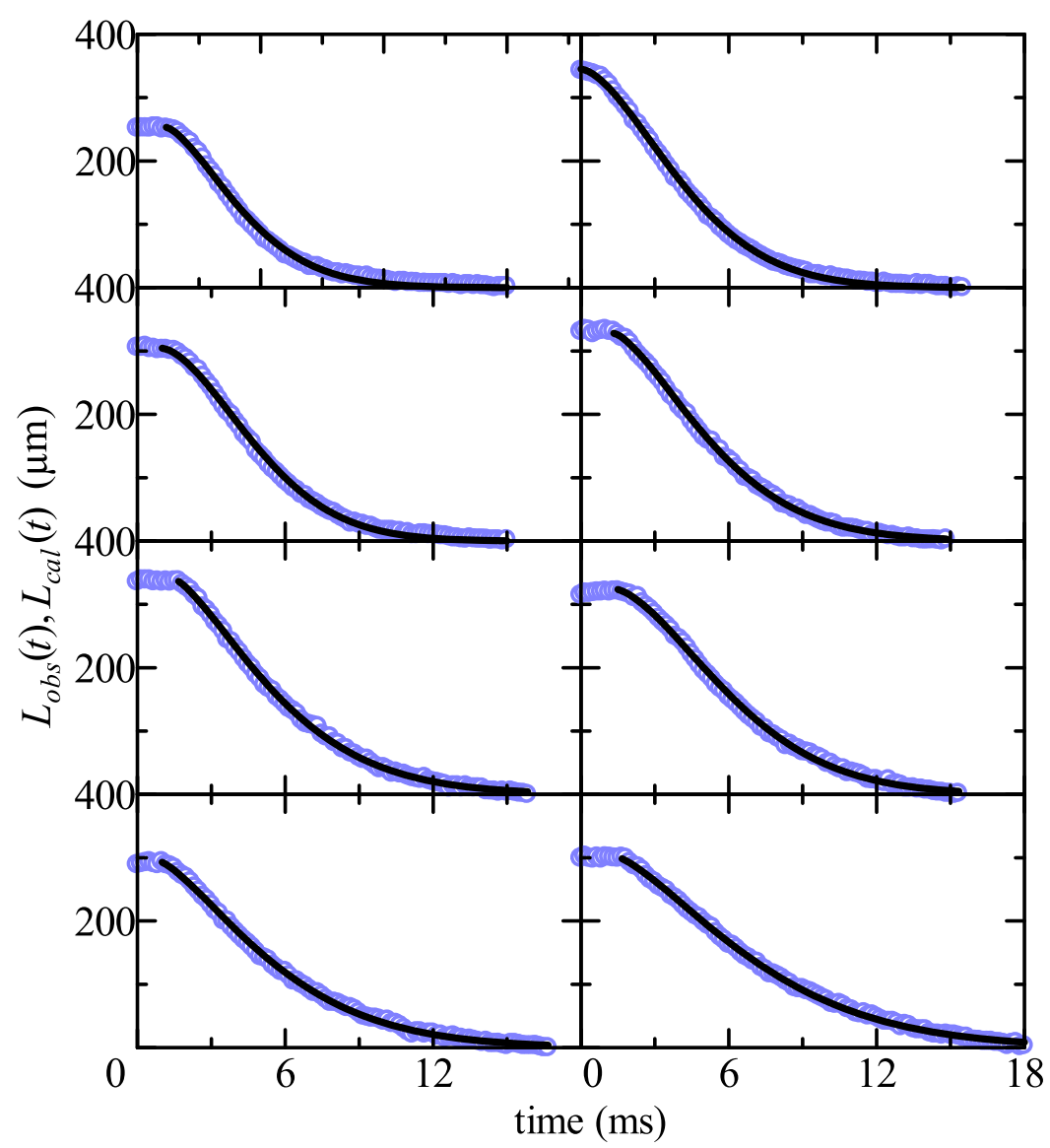

Figure 8 Plots of $L_{o b s}(t)$ as a function of time for several Vorticella sp. The solid line is a fit to a stretched exponential function (Eq. (8)).

Table 2 Fitting parameters of a stretched exponential function (Eq. (8))

\begin{tabular}{ccccccc}
\hline Data & $c(\mu \mathrm{m})$ & $\beta$ & $t_{0}{ }^{\prime}(\mathrm{ms})$ & $\tau_{S}(\mathrm{~ms})$ & $\chi^{2}$ & $u_{\max }(\mathrm{cm} / \mathrm{s})$ \\
\hline 1 & $2.54 \times 10^{2}$ & 1.54 & 1.12 & 3.82 & $2.2 \times 10^{-5}$ & 5.00 \\
2 & $3.45 \times 10^{2}$ & 1.64 & $-8.85 \times 10^{-3}$ & 4.95 & $7.8 \times 10^{26}$ & 5.36 \\
3 & $3.05 \times 10^{2}$ & 1.71 & $9.17 \times 10^{-1}$ & 4.76 & $1.3 \times 10^{-5}$ & 5.02 \\
4 & $3.28 \times 10^{2}$ & 1.49 & 1.31 & 4.84 & $4.9 \times 10^{-6}$ & 5.04 \\
5 & $3.40 \times 10^{2}$ & 1.41 & 1.46 & 5.04 & $6.4 \times 10^{-6}$ & 4.97 \\
6 & $3.24 \times 10^{2}$ & 1.61 & 1.34 & 5.73 & $1.0 \times 10^{-5}$ & 4.31 \\
7 & $2.94 \times 10^{2}$ & 1.38 & $8.90 \times 10^{-1}$ & 5.49 & $7.5 \times 10^{-6}$ & 3.93 \\
8 & $3.01 \times 10^{2}$ & 1.39 & 1.41 & 6.67 & $7.7 \times 10^{-6}$ & 3.32 \\
\hline
\end{tabular}

criminate between Eq. (4) and Eq. (8) more as to better fit of data, the following $\chi^{2}$-map method was applied ${ }^{17}$. Select two parameters and calculate the value of $\chi^{2}$ as the value of the parameters is changed independently. We consider an orthogonal coordinate system, $\mathrm{O}-\mathrm{XYZ}$, and the two parameters of a fitting function are selected as $\mathrm{X}$ and $\mathrm{Y}$ coordinate axes and the calculated $1 / \chi^{2}$ is plotted on the $Z$ coordinate one. The value of $1 / \chi^{2}$ approaches infinitely when a function fits well to the measured data. Such three-dimension graph is named a ' $\chi^{2}$-map'. This method enables us to estimate the fitting properties in the vicinity of a stable point in a trial function (fitting function). Figure 9 shows the $\chi^{2}$-maps for Eq. (4) and Eq. (8) applied to $L_{o b s}(t)$. The most considerable fitting parameters of Eq. (4) should be $p$ and $q$, while those of Eq. (8) are $\tau_{S}$ and $\beta$. In case of Eq. (8) a unique peak is observed in a $\chi^{2}$-map and it means that the fitting parameters $\tau$ and $\beta$ are independent for the fit. On the other hand, the $\chi^{2}$-map of Eq. (4) has a wall shape with the values of $1 / \chi^{2}$ themselves low and it can be presumed that there is a strong correlation between $p$ and $q$. This implies that it is difficult to separate $p$ and $q$ in Eq. (4) for the fit to the experimental data. The $\chi^{2}$-map method indicates that a stretched exponential is more suitable to explain the concentration process of Vorticella sp. than a double exponential function.

The $\beta$ value of Eq. (8) ranges from 1.38 to 1.71 as listed in Table 2 . In case of $1 \leq \beta$, a stretched exponential function was deduced by Avrami to explain the crystallization kinetics that the crystal particles appear as nuclei and grow with the 

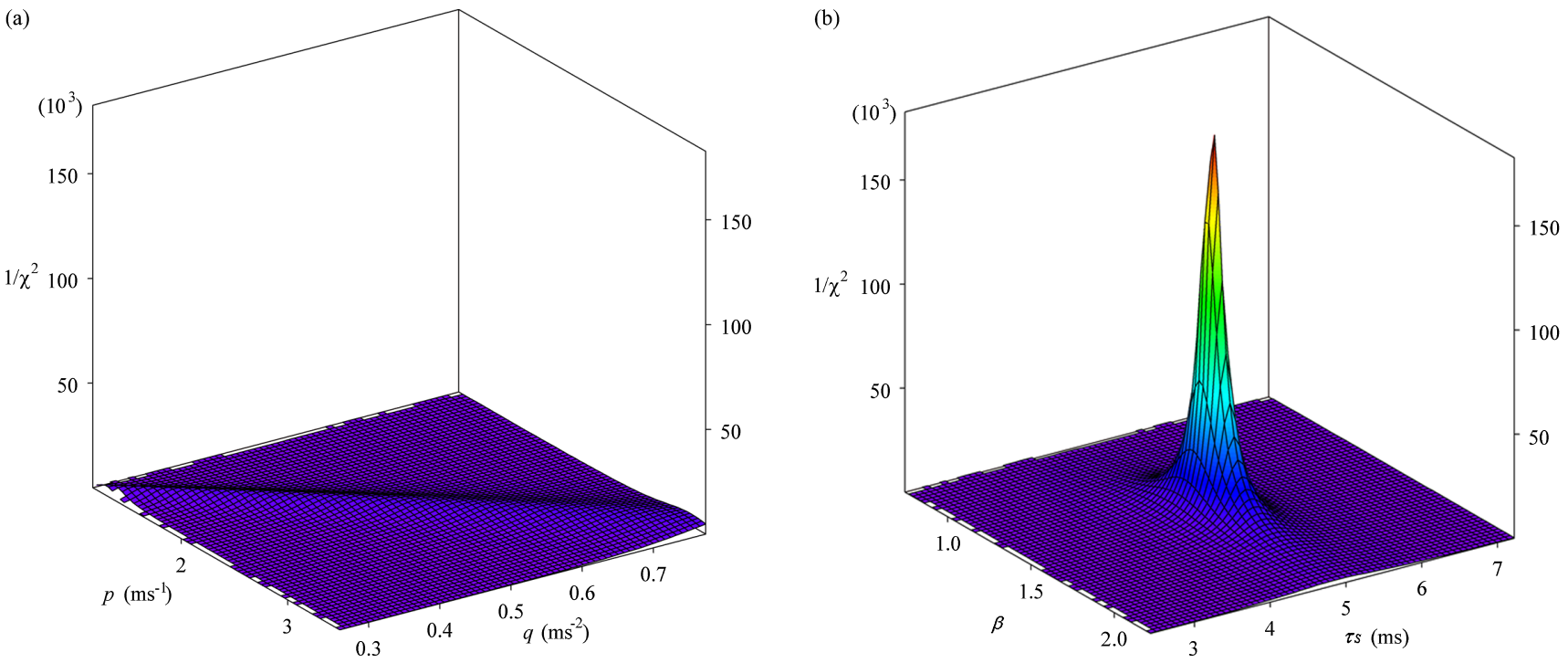

Figure $9 \chi^{2}$-maps for a double exponential function (Eq. (4)) (a) and a stretched exponential function (Eq. (8)) (b).

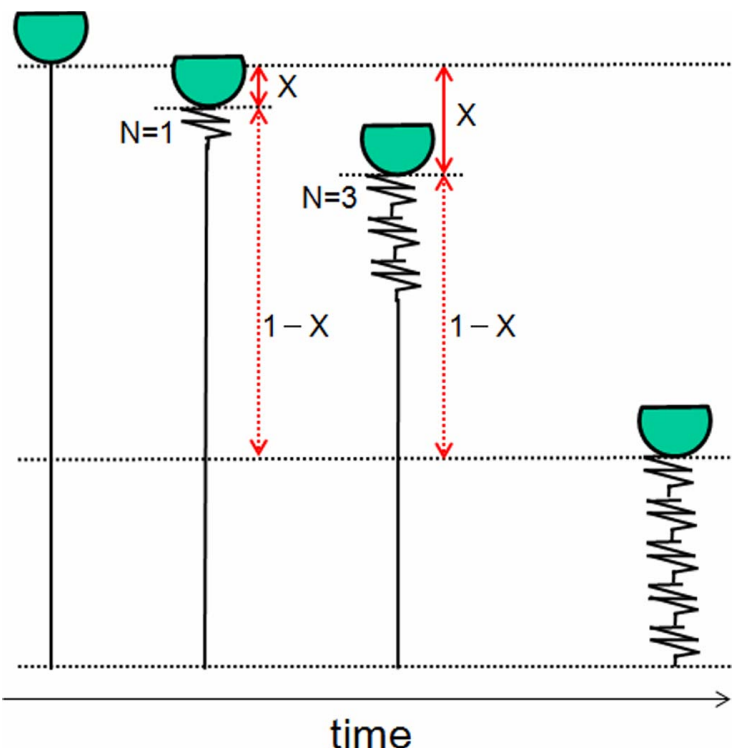

Figure 10 Schematic image of the contraction mechanism of Vorticella sp. based on the nucleation and growth model

particle impingement ${ }^{16}$. Such nucleation and growth model is applied to the contraction process of Vorticella $\mathrm{sp}$. as follows.

Figure 10 shows the contractile process model of Vorticella sp. which is composed of a zooid as a bell and a spasmoneme as a line. The spasmoneme consists of small segments and each segment contracts as a nucleus appears and grows. The nucleation starts at the zooid and spreads downwards along the spasmoneme in a one-dimensional space. The number of nuclei at a time $t, N$, is assumed to be explained as

$$
N=z t^{\alpha},
$$

where $\alpha$ is space dimension from 0 to 1 . If the reduced length owing to the contraction at a time $t$ is $X$ and the full length for the contraction is unity, the stalk length is $1-X$. $X$ increases with time and the increment is assumed to be proportional to $N$ and $(1-X)$ as

$$
\frac{d X}{d t}=A(1-X) N
$$

Combining Eq. (11) and Eq. (12), one obtains, for the stalk length, $1-X$, which is proportional to the temporal change in the reduced length, $L_{c a l}(t)$,

$$
L_{c a l}(t) \propto 1-X=\exp \left(-A z t^{\alpha}\right), \beta=\alpha+1 .
$$

The experimentally evaluated $\beta$ value ranges from 1.38 to 1.71 as listed in Table 2. It indicates that the space dimension for the contraction, $\alpha$ ranges from 0.38 to 0.71 (the average over eight cells: 0.52 ). The fact that the experimentally evaluated space dimension, $\alpha$, is smaller than unity supports the contraction model in which the segments of the spasmoneme are aligned one-dimension. The nucleation and growth model expresses the practical contraction profile that the coiling of the stalk starts near the zooid and spread downwards in a one-dimensional space. This image accords with the fact that the coiling of the stalk starts near the zooid and spreads downwards suggests that some stimulus runs down the stalk as shown in Figure 4.

\section{Conclusion}

In this report, we executed two kinds of fitting functions, a double exponential function and a stretched exponential function for the entire contraction process of living Vorticella sp.. A double exponential function is deduced from a conventional damped harmonic oscillator model, while a stretched exponential is newly proposed to express a more 
particularly observed contraction mechanism in which the contraction of the stalk starts near the zooid and spreads downwards along the stalk. The index value of the stretched exponential is experimentally evaluated as 1.38-1.71 and consistent with the model in which the contraction occurs like nucleation and growth in a one-dimensional space. The space dimension for the nucleation is obtained as $0.38-0.71$ by subtracting one from the index value and would be related to the propagation of some stimuli for the contraction. The usefulness of a stretched exponential function is also supported by the $\chi^{2}$ map method and the fitness of the stretched exponential to the experimental data is better than that of the double exponential function.

\section{References}

1. Nagai, M., Kumemura, M., Asai, H. \& Fujita, H. Binding of Artificial Object to Vorticella for a Microsystem Powered by a Microorganism. e-J. Surf. Sci. Nanotech. 7, 673-676 (2009).

2. Huck, W. T.S. Responsive polymers for nanoscale actuation. Materials today 11, 24-32 (2008).

3. Hoffmann-Berling, H. Der mechanismus eines neuen, von der muskelkontraktion verschiedenen kontraktionszyklus. Biochim. Biophys. Acta 27, 247-255 (1958).

4. Weis-Fogh, T. \& Amos, W. B. Evidence for a new mechanism of cell motility. Nature 236, 301-304 (1972).

5. Amos, W. B. Structure and Coiling of the Stalk in the Peritrich Ciliates Vorticella and Carchesium. J. Cell Sci. 10, 95-122 (1972).

6. Asai, H. $\mathrm{Ca}^{2+}$-driven contraction of spasmoneme in Vorticellidae. Jpn. J. Protozool. 38, 133-152 (2005).

7. Jones, A. R., Jahn, T. L. \& Fonseca, J. R. Contraction of protoplasm. IV. Cinematographic analysis of the contraction of some peritrichs. J. Cell. Physiol. 75, 9-19 (1970).

8. Moriyama, Y., Hiyama, S. \& Asai, H. High-Speed Video Cinematographic Demonstration of Stalk and Zooid Contraction of Vorticella convallaria. Biophys. J. 74, 487-491 (1998).
9. Upadhyaya, A., Baraban, M., Wong, J., Matsudaira, P., van Oudenaarden, A. \& Mahadevan, L. Power-Limited Contraction Dynamics of Vorticella convallaria: An Ultrafast Biological Spring. Biophys. J. 94, 265-272 (2008).

10. Misra, G., Dickinson, R.B. \& Ladd, A.J.C. Mechanics of Vorticella Contraction. Biophys. J. 98, 2923-2932 (2010).

11. Kamiguri, J., Tsuchiya, N., Hidema, R., Yatabe, Z., Shoji, M., Hashimoto, C., Pansu, R. B. \& Ushiki, H. Contraction Behaviors of Vorticella sp. Stalk Investigated using High-Speed Video Camera. II: Viscosity Effect of Several Types of Polymer Additives. Biophysics 8, 11-19 (2012).

12. Ushiki, H. Fluorescence Probes in Polymers and Liquid Crystals-Complex Macromolecular Chain Dynamics - Proposal from Far East-. In Applied Fluorescence in Chemistry Biology and Medicine. (Rettig, W., Strehmel, B., Schrader, S. \& Seifert, H. eds.) pp. 325-370 (Springer, New York, 1999).

13. Nakagawa, T. \& Oyanagi, Y. Tokyo Daigaku Shuppan-kai (Tokyo Daigaku Shuppan-kai, Japan, 1982).

14. Savitzky, A. \& Golay, M. J.E. Smoothing and Differentiation of Data by Simplified Least Squares Procedures. Anal. Chem. 36, 1627-1639 (1964).; Bromba, M.U.A. \& Ziegler, H. Efficient computation of polynomial smoothing digital filters. Anal. Chem, 51, 1760-1762 (1979).; Minami, S. Kagaku keisoku notameno hakei data shori -Keisoku system niokeru maikon pasokon katsuyou gijutu (CQ Shuppan, Japan 1986).

15. Kohlrausch, R. Theorie des elektrischen Rückstandes in der Leidener Flasche. Ann. Phys. 167, 56-82 (1854).; Kohlrausch, R. Theorie des elektrischen Rückstandes in der Leidener Flasche. Ann. Phys. 167, 179-214 (1854); Williams, G. \& Watts, D. C. Non-symmetrical dielectric relaxation behaviour arising from a simple empirical decay function. Trans. Faraday Soc. 66, 80-85 (1970).

16. Avrami, M. Kinetics of Phase Change. I General Theory. $J$. Chem. Phys. 7, 1103-1112 (1939).

17. Hashimoto, C., Rouch, J., Lachaise, J., Graciaa, A. \& Ushiki, H. Complex macromolecular dynamics: I. Estimation technique for time-resolved emission anisotropy ratio of chromophores incorporated into polymer chains. Eur. Polym. J. 40, 19972008 (2004). 